\title{
I Gilets Jaunes. La Francia popolare in rivolta
}

\author{
di Antimo Luigi Farro
}

\section{Online e offline}

Le mobilitazioni dei Gilets Jaunes prendono avvio online con post, video e altri messaggi di cittadini che, nel mese di ottobre 2018, comunicano tra loro denunciando e opponendosi fermamente all'introduzione in Francia di una nuova tassa sul consumo di gasolio al distributore. Una misura fiscale che, per il governo francese, dovrebbe andare in direzione della tutela ambientale. Secondo la visione governativa, la nuova tassa dovrebbe, infatti, scoraggiare il consumo di un carburante valutato sul piano scientifico come particolarmente inquinante e, in questo modo, contribuire all'attuazione di politiche di protezione ambientale, politiche da implementare nell'ambito di interventi da svolgere sul piano globale, al fine di fronteggiare la crisi ecologica che attanaglia il pianeta.

La nuova tassa rappresenta invece per parte dei cittadini del Paese un onere economico aggiuntivo, destinato a incidere in termini significativi sui budget, individuali o familiari, di automobilisti, camionisti e altri utilizzatori abituali di veicoli con motori diesel. Tuttavia le mobilitazioni dei Gilets Jaunes non sono complessivamente improntate alla critica delle politiche di salvaguardia ambientale. Le loro critiche si concentrano, infatti, sulla politica fiscale del governo che, con questa tassa, aggrava ulteriormente la difficile condizione sociale di parte degli strati più bassi della classe media ("la petite classe moyenne") e di strati popolari. Una questione che, secondo queste critiche, le élite del potere non prendono in considerazione allorquando elaborano o sostengono politiche di breve o di lungo periodo, come quelle della salvaguardia ambientale.

I Gilets Jaunes individuano queste élite innanzitutto negli attori politici di governo, a cominciare dal presidente della Repubblica Emmanuel Macron, 
e negli alti funzionari dei ministeri, costituiti principalmente da personale formato nelle "Grandes Écoles". Istituzioni che hanno per originario e principale compito la preparazione di alti dirigenti dell'apparato di Stato o di differenti settori dell'intervento pubblico, di professori dell'insegnamento superiore e di specialisti della ricerca scientifica ${ }^{1}$.

Il che conferisce generalmente, ma non senza controversie interpretative, ai dirigenti e ad altri professionisti formati nelle "Grandes Écoles", soprattutto in quelle considerate di maggiore rilievo come l'École Polytechnique e l'Ena (Ecole Nationale de l'Administration), l'immagine di cittadini che, visti gli esclusivi criteri meritocratici con cui sono selezionati, meritano di ricoprire posizioni di rilievo nell'amministrazione statale o in altri contesti organizzativi del settore pubblico. Un'immagine contestata nell'ambito delle mobilitazioni dei Gilets Jaunes, da cui emergono considerazioni critiche espresse nei confronti di questi dirigenti, visti come dispensatori di impropri giudizi disprezzanti chi "non c'è l'ha fatta", chi non è riuscito, pur avendone, in teoria, la possibilità - come tutti i cittadini della Repubblica- di raggiungere una collocazione sociale ed economica di rilievo o almeno sodisfacente. Giudizi disprezzanti che attori delle mobilitazioni ravvisano come manifestazione di un disprezzo espresso da membri delle élite nei confronti di chi, come altri, avrebbe potuto "farcela" se avesse tentato o vi hanno tentato senza riuscirci. Un "mépris" che nelle fila dei Gilets Jaunes viene avvertito in comportamenti ed espressioni verbali di denigrazione dovute a membri di queste stesse élite. Denigrazioni di cui questi attori riscontrano le prove anche in dichiarazioni e discorsi tenuti dal presidente della Repubblica, che, dal canto suo, ha ricevuto la sua formazione professionale in "Grandes Ecoles". Un disprezzo del capo dello Stato verso le persone ritenute socialmente perdenti che, per i Gilets Jaunes, si manifesta in maniera esemplare con la frase: «les gens qui réussissent et les gens qui ne sont rien». Frase pronunciata dal presidente Macron, e reperibile col sonoro di un video presente online, in cui si vede lo stesso capo dello Stato che, riferendosi alle persone presenti in una stazione ferroviaria, le distingue in due gruppi: un primo gruppo, di cui pro-

${ }^{1}$ L'accesso a queste "Grandes Écoles" richiede una preparazione particolare, che i candidati perseguono frequentando, per periodi significativi, le "classes préparatoires" al fine di poter affrontare con successo il concorso di ingresso, particolarmente selettivo, a queste istituzioni formative. L'uscita, dopo il periodo di formazione, da una di queste istituzioni avviene generalmente attraverso graduatorie, che conferiscono alla prima o al primo classificato, e a coloro che immediatamente li seguono, le collocazioni professionali migliori, di maggiore prestigio e potere, nell'ambito delle istituzioni o delle altre organizzazioni in cui sono inseriti (P. Bourdieu, M. de Saint Martin, 1989, La Noblesse d'État. Grandes écoles et esprit de corps, Minuit, Paris). 
pone un'immagine fortemente positiva, costituito da coloro che sono riusciti - con proprie capacità, impegno e sforzo - ad affermarsi nella vita sociale e un secondo, presentato molto negativamente, formato invece da quanti, definiti come il niente, non vi sono riusciti o non hanno nemmeno tentato ${ }^{2}$.

\section{Denuncia delle élite}

La denuncia dell'elitismo che manifesta disprezzo nei confronti di singoli e gruppi ritenuti come il niente, costituisce una componente essenziale del discorso contestativo dei Gilets Jaunes. Una componente che si connota come critica culturale esplicitata contro visioni del mondo, proprie delle élite che, nel predisporre progetti di sviluppo o di modernizzazione attraverso cui esercitano il loro potere, non contemplano l'esistenza di altre visioni del mondo e l'impatto socio-economico che la messa in pratica delle loro iniziative progettuali comporta per le condizioni di vita di strati popolari e di parti di classe media.

Le mobilitazioni denunciano le élite politiche o tecnocratiche e, principalmente, i loro membri insediati in tecnostrutture ${ }^{3}$ che hanno sede nella

${ }^{2}$ Il presidente Emmanuel Macron pronuncia questa frase nell'estate 2017, in occasione dell'inaugurazione di una nuova struttura situata in una stazione ferroviaria parigina dove, come in altri luoghi similari, circolano, senza incontrarsi, persone di differente posizione sociale.

3 Seguendo in termini critici la concettualizzazione proposta da J.K. Galbraith per definire gli attori dirigenti del nuovo Stato industriale (The New Industrial State, Houghton Mifflin, Boston, 1967), col termine tecnostruttura qui si intende designare due tipi di raggruppamenti. Un primo costituito da agenti che mobilizzano, tramite azioni svolte online e offline, risorse tecnologiche, economiche e scientifiche per definire progetti e obiettivi con il cui perseguimento si alimenta il potere delle forze sistemiche e il loro dominio sugli assetti sociali del XXI secolo. Si tratta di forze sistemiche di rilievo planetario come lo sono quelle del capitale finanziario che operano sul piano del globo senza essere esposte a controlli istituzionali - di Stati nazionali o di organismi politici sovranazionali come l'Unione Europea - e senza rapporto con l'economia reale. Un secondo tipo di raggruppamento include tecnostrutture che operano in contesti istituzionali, come gli apparati ministeriali, per sostenere l'azione del governo di un Paese o di un altro territorio politicamente delimitato. Si tratta di esercizi di potere che questi differenti tipi di tecnostrutture compiono predisponendo modelli di condizionamento della vita di individui e gruppi, dell'evoluzione dei loro agire e della loro stessa esistenza. Individui e gruppi che, a loro volta, vivono esperienze soggettive e intersoggettive non riconducibili a semplici esecuzioni pratiche dei modelli di agire loro imposti. Esperienze che comportano la creazione di contrasti tra queste strutture di potere e soggetti individuali o membri di gruppi che puntano a essere controllori dell'evoluzione della propria esistenza di singoli (A.L. Farro, D.G. Demirhisar, 2014, «The Gezi Park Movement A Turkish Experience of the 21st Century Collective Movements», International Revue of Sociology, 24, 1, March, pp. 176-189) e della creazione di proprie socialità e loro socializzazioni (U. Beck, 2006, Cosmopolitan Vision, 
capitale, ritenendole come principali portatrici di queste visioni culturali elitiste che fungono da matrice per la formulazione di politiche, come quelle di protezione ambientale, che non contemplano i problemi sociali ed economici di una parte rilevante dei cittadini del Paese.

Una presa di posizione anti-élite la cui matrice sociale e culturale è elaborata dai Gilets Jaunes nel corso dei dibattiti che hanno luogo sui social ed è proposta online con espressioni che evocano le difficili condizioni sociali in cui versa una parte della popolazione. Dibattiti in cui intervengono, oltre a Gilets Juanes o loro sostenitori, anche altri gruppi o "comunità di attori" e agenti politici ${ }^{4}$. Dibattiti in cui emergono propositi che - provenienti dall'ambito de-

Polity Press, Cambridge; A. Touraine, 2007, A New Paradigm. For Understanding Today's World, Polity Press, Malden; M. Castells, 2012, Networks of Outrage and Hope: Social Movements in the Internet Age, Polity Press, Cambridge-Maiden).

${ }^{4}$ I dibattiti che si svolgono online in francese su scala mondiale in merito all'azione collettiva dei Gilets Jaunes sono aggetto di una ricerca effettuata nel corso delle prime settimane di mobilitazione offline (dal 13 novembre al 19 dicembre 2018) da Alto Data Analytics, azienda, specializzata del settore. L'analisi di Alto si concentra in particolare sulla comprensione del ruolo che ricoprono e l'influenza che, sul piano digitale, esercitano personale politico, società civile, social e piattaforme dei digital media nel costruire il dibattito pubblico sulle mobilitazioni dei Gilets Jaunes. La ricerca prende in considerazione dati pubblici relativi a 11.510.953 risultati dovuti a 1.053 .427 autori che conducono 538.269 conversazioni attraverso piattaforme di social e digital media quali Twitter, Facebook, YouTube, Instagram, blog, forum e altre comunità digitali, come anche gli utenti che commentano le informazioni dei media che coprono i primi sviluppi delle mobilitazioni dei Gilets Jaunes. In primo luogo la ricerca sottolinea il fatto che il dibattito online risulta influenzato da un'esigua minoranza di utenti. Media digitali emergenti in Francia e altri non francesi, come in particolare i russi, di orientamento governativo, Russia Today e Sputnik, ricoprono un ruolo centrale nell'orientare il dibattito, introducendo narrative chiave e creando contenuti. In questo contesto però guadagnano visibilità anche Facebook, utilizzato come cruciale strumento di coordinazione del dibattito online, e Twitter che interviene in quest'ultimo promuovendo misure di antidisinformazione. Si tratta di un dibattito che si sviluppa a opera di cinque distinte comunità, individuate attraverso l'applicazione di modelli analitici e di tecniche prodotte o adottate dagli studiosi di Alto. Ognuna di queste comunità include aggregazioni di utenti direttamente impegnati nelle mobilitazioni o interessati a definirne i rilievo che assumono rispetto agli assetti politici del Paese o alla strutturazione della sua vita sociale. La prima comunità, denominata Sostenitrice dei Gilets Jaunes e costituita dal 58\% degli autori, raggruppa principalmente cittadini che mostrano la loro distanza rispetto alle ideologie politiche tradizionali, affrontano la questione della violenza della polizia e criticano il governo adottando complessivamente un tono satirico. La seconda comunità, dovuta al 15,3\% degli autori e denominata Sostenitori di Macron, empatizza con le mobilitazioni ma allo stesso tempo supporta il governo. È inoltre solidale con le fasi iniziali dell'azione, ma ne critica successivi sviluppi in cui si hanno violenze dovute a gruppi di destra e di sinistra. La terza comunità, denominata Iniziativa Gilets Jaunes e dovuta al 13,8\% degli autori, si concentra sulle denunce sull'aumento del carburante, sulla violenza della polizia nel corso delle manifestazioni e definisce le mobilitazioni non politiche. Leader di La France Insoumise (organizzazione politica di sinistra radicale che ha 
gli stessi Gilets Jaunes e facendo riferimento alla nuova tassa sul carburante promossa dalle élite al potere ritenute insensibili rispetto alla situazione socioeconomica di parte significativa dei cittadini - suonano nel modo seguente: "Les élite parlent de fin du monde, quand nous, on parle de fin du mois».

\section{Un'azione collettiva}

Fin dal loro avvio online, le mobilitazioni si presentano così come partecipi di un'azione collettiva ${ }^{5}$ anti-élite. Élite individuate non solo in quelle, relativamente visibili, costitutive di apparati di direzione politica del Paese, di tecnostrutture di aziende e di altre organizzazioni pubbliche. Ma anche in quelle, non immediatamente percepibili, costituite da detentori di capitale finanziario e da dirigenti di tecnostrutture che ne indirizzano i flussi su scala planetaria, senza essere sottoposti a controlli istituzionali e senza intervenire nell'economia reale. La politica del governo in carica e l'azione dello stesso presidente della Repubblica, sono considerate nelle fila dei Gilets Jaunes come promotrici di politiche economiche sostenitrici di questi interessi finanziari e non dello sviluppo dell'economia reale che è invece da supportare sostenendo le attività industriali, scientifiche, tecnologiche e dei servizi che incrementano o creano occupazione.

A tale riguardo è contestata soprattutto la strutturazione complessiva della società nazionale, strutturazione intesa, presso gli stessi Gilets Jaunes, come un assetto di rapporti sociali in cui prevalgono dominazioni e vantaggi di cui

come esponente principale Jean-Luc Mélenchon) e di Génération, le mouvement (il cui leader Benoît Hamon proviene dal PS, Parti Socialiste) sono parte di questa comunità che si oppone decisamente alla Comunità di destra. La quarta comunità è costituita proprio dalla Comunità di destra che, composta dall' $8,3 \%$ degli autori, si riferisce alle mobilitazioni dei Gilets Jaunes per criticare il governo e condannare la sinistra per gli atti di violenza che hanno luogo nel corso delle manifestazioni. La quinta comunità, denominata Nuovi Media, comprende il 7,9\% degli autori, i quali intervengono con abilità nei dibattiti delle altre comunità con loro messaggi (Alto Data Analytics, 2018, Public Digital Sphere Analysis of Yellow Vests Movement, Madrid, New York Office, São Paulo, www.alto-analytics.com/en_US/).

${ }_{5}$ Per azione collettiva qui si intende la condotta svolta in comune da attori che si situano collettivamente in rapporti sociali non paritari, rapporti che riguardano il controllo degli orientamenti della vita sociale. Rapporti in cui gli attori o le forze dirigenti esercitano domini sociali dei quali risentono gli attori che vi si oppongono puntando per un verso a perseguire alternative a questo stesso dominio e, per un altro, a contendere agli stessi attori e forze dominanti il controllo della produzione e della diffusione delle informazioni relative alla strutturazione dei diversi campi della vita sociale (cfr. A.L. Farro, 2000, Les mouvements sociaux. Diversité, action collective et globalisation, Les Presses de l'Université de Montréal, Montreal, pp. 159-166). 
sono depositari da un lato le élite formate nelle Grandes Ecoles e inserite nelle istituzioni $o$ in tecnostrutture insediate principalmente nella capitale e, dall'altro, le forze del grande capitale e della finanza di rilievo planetario.

Le iniziative dei Gilets Jaunes, fin dall'iniziale azione condotta online, si declinano opponendosi a questo assetto di rapporti sociali e hanno come obiettivi prioritari la difesa e il sostegno delle condizioni sociali di cittadini de la "petite classe moyenne" e degli strati popolari, a cominciare da quelli i cui insediamenti abitativi sono situati in zone delle estreme periferie di grandi aree metropolitane $o$ in altre lontane da centri urbani dotati di strutture commerciali e di servizi. Zone spesso non servite da trasporti pubblici, il che rende necessario il ricorso a mezzi di trasporto privati, con gli oneri economici che questo comporta.

Le stesse prime mobilitazioni critiche condotte online hanno del resto origine dal vissuto di cittadini che per ragioni lavorative, per accedere a servizi, per accompagnare i figli a scuola o per altri motivi ancora percorrono distanze di varia grandezza utilizzando veicoli alimentati a gasolio. L'onere economico che questo comporta risulta ancora di maggiore rilievo per quanti utilizzano veicoli con motori diesel per svolgere il proprio lavoro.

Un primo post sull'argomento viene proposto il 10 ottobre 2018 proprio da un camionista della regione parigina, che, abitualmente, percorre con il suo automezzo un numero rilevante di chilometri per caricare merci o per consegnarle. Si tratta di un messaggio online di denuncia di un fiscalismo ritenuto socialmente iniquo, ma anche un messaggio dovuto a una persona destinata a diventare una figura simbolo del movimento, senza esserne un dirigente o leader organizzativamente riconosciuto.

Passano meno di una decina di giorni dalla diffusione di questo messaggio e un breve video, dedicato ai costi aggiuntivi dovuti alla nuova tassazione, comincia a essere scaricato attraverso diversi contatti stabiliti via internet, fino a raggiungere, a fine dicembre dello stesso anno, oltre sei milioni di contatti. Il video è stato inviato online da una signora bretone poi divenuta, a sua volta, una delle figure simbolo del movimento. Una petizione lanciata online da una giovane imprenditrice precaria - insediata a Parigi e anch'essa destinata a divenire una figura simbolo del movimento- per chiedere la riduzione del costo del carburante viene, dal canto suo, sottoscritta rapidamente da un numero crescente di persone, fino a superare il milione di adesioni nel mese di dicembre.

I contatti online tra singoli soggetti rappresentano in quest'ultimo caso, come negli altri visti in precedenza, una componente rilevante della costruzione e dello sviluppo dell'azione collettiva. Contatti che danno luogo anche a raggruppamenti che si costituiscono autonomamente l'uno dall'altro e re- 
stano, allo stesso tempo, in rapporto tra loro nel promuovere o partecipare a diverse iniziative online.

Questi gruppi di singoli soggetti, costituiti prevalentemente online e in autonomia tra di loro, danno luogo a confronti e dibattiti in cui i temi fiscali sollevati con l'opposizione alla nuova tassa sul carburante, sono riconsiderati nell'ambito di questioni di ordine amministrativo, socio-economico e politico trattate nel corso dell'evolversi delle mobilitazioni.

Le questioni di ordine amministrativo riguardano la definizione stessa di normative di regolazione della vita sociale, come in particolare quelle che comportano sanzioni economiche imposte ai cittadini per non aver rispettato norme del codice della strada ritenute inapplicabili dagli stessi automobilisti $^{6}$. Le questioni di ordine socio-economico riguardano, a loro volta, le condizioni sociali e i bassi livelli di reddito di parte significativa dei cittadini, dovuta, per i Gilets Jaunes, al potere esercitato sul piano sociale ed economico dai detentori di grandi capitali. Il che conduce gli attori delle mobilitazioni a rivendicare la realizzazione di un'effettiva giustizia sociale, da alimentare anche attraverso la revisione delle regole di prelievo fiscale a vantaggio dei redditi bassi e medio bassi, come anche un aumento del salario minimo, lo Smic, il Salaire minimum interprofessionnel de croissance, che ammonta al momento delle prime mobilitazioni a 1498,50€ mensili lordi e $1.188 €$ netti. Le questioni di ordine politico sollevate dai Gilets Jaunes, infine, riguardano principalmente la denuncia dell'accentramento del potere negli organismi istituzionali vigenti e la rivendicazione di nuove forme di democrazia diretta, anche di tipo referendario.

\section{Manifestazioni}

Le questioni introdotte progressivamente nel corso delle comunicazioni online assumono particolare rilievo e presentano ulteriori sviluppi con l'insorgere di altre iniziative destinate a rendere particolarmente visibile l'azione conflittuale dei Gilets Jaunes. La comunicazione online diventa, a sua

${ }^{6}$ Ne rappresenta un esempio concreto la riduzione, stabilita nel luglio 2018, dei limiti di velocità da 90 a $80 \mathrm{~km}$ orari sulle strade extra-urbane, prive di spartitraffico tra le due carreggiate. Una misura dettata da esigenze di migliorare la sicurezza stradale che comporta la promulgazione di multe per eccesso di velocità a diversi automobilisti che, abituati al rispetto del divieto precedente e residenti in aree prive o con scarsa presenza di trasporti pubblici, sono obbligati ricorrere a propri mezzi per raggiungere dalle loro abitazioni luoghi di lavoro, scuole dei figli, installazioni commerciali, sedi di amministrazioni e di servizi vari sovente distanti decine di chilometri. 
volta, parte costitutiva dell' organizzazione e della diffusione di informazioni riguardanti queste iniziative, rappresentate principalmente da manifestazioni svolte cortei e testimonianze espressive, anche di carattere artistico, che, a partire dal sabato 17 novembre 2018 si tengono durante i weekend a Parigi $\mathrm{o}$ in centri cittadini di altre aree metropolitane e in altri contesti urbani di varia dimensione. A queste mobilitazioni dal forte impatto mediatico, si aggiungono altre forme di azione svolte in differenti contesti territoriali, anche con occupazioni temporanee di spazi viari e snodi o tratti autostradali. Altre iniziative ancora si concretizzano con l'installazione di presidi che si vogliono permanenti in aree in cui sono collocati "ronds-points", rotatorie di regolazione del traffico, dove gruppi di Gilets Jaunes fungono da polo di attrazione per loro simpatizzanti o sostenitori, si preoccupano di fornire informazioni sul senso che danno alla loro lotta a quanti sono interessati a riceverle e, talvolta, si attribuiscono il ruolo di informatori aggressivi e di regolatori della circolazione.

Le grandi manifestazioni tenute con scadenza settimanale sono avviate con l'iniziativa definita Atto I dell'azione dei Gilets Jaunes, Atto che comporta in tutta la Francia, secondo fonti ministeriali, la partecipazione di 282.710 manifestanti e di 2.034 luoghi dove si registra la loro presenza.

A Parigi, in questa fase iniziale, si hanno a metà giornata 29 manifestazioni con una partecipazione di circa 1.200 persone. Nel pomeriggio se ne contano meno di una ventina e il loro numero continua a decrescere verso sera. In tarda serata, si segnala ancora la presenza di circa 400 persone nella vasta area centrale dove sorgono la Place de la Concorde, l'Avenue des Champs Élysées e la Place de L'Etoile. Piazza, con quest'ultima, che ha al suo centro lo storico Arc de Triomphe, destinato a divenire un luogo altamente simbolico delle proteste dei Gilets Jaunes. La manifestazione infine si scioglie tra tensioni che si stabiliscono tra Gilets Jaunes e forze dell'ordine presenti sul posto.

Nelle altre parti del Paese si hanno mobilitazioni di rilievo a Bordeaux, capoluogo della regione Nouvelle-Aquitaine, con la partecipazione di qualche centinaio di Gilets Jaunes che si concentrano soprattutto nell'area della centrale Place de la Republique. Nella stessa regione si segnala la presenza di diverse manifestazioni e presidi. Una sessantina, con la partecipazione di circa 7.000 persone, hanno luogo in vari punti dei tracciatati autostradali, interessando la A10 e la A63, dove, in particolare, si assiste a una concentrazione dei manifestanti nell'area del Pont D'Aquitaine, luogo da cui si dirama il collegamento con l'autostrada che porta a Parigi, e dove qualche centinaio di Gilets Jaunes intralcia lo scorrimento del traffico nel corso di tutto il weekend. Altre manifestazioni ancora che si hanno nella stessa regione, 
fanno registrare una partecipazione che varia dalle 300 alle 2.000 persone che si mobilizzano in località come Arcachon, Libourne, Pont d'Aquitaine, Médoc e Langon. Nell'area del Vaucluse, Dipartimento della Regione Provenza-Alpi-Costa Azzurra, circa 3000 persone partecipano alle mobilitazioni e si annoverano 43 aree di blocco o di rallentamento del traffico. A Marsiglia, capoluogo del Dipartimento delle Bouches-du-Rhône e della Regione Provenza-Alpi-Costa-Azzurra, le mobilitazioni si traducono principalmente nel blocco o nel rallentamento del traffico di importanti snodi stradali. A Montpellier, capoluogo della Regione Languedoc-Roussillon, si assiste a manifestazioni a cui partecipano 10.000 persone e a blocchi o rallentamenti del traffico. A Perpignan, capoluogo del Dipartimento dei Pirenei orientali situato nella Regione denominata Occitania, si registrano principalmente blocchi del traffico a caselli autostradali e rallentamenti della circolazione alla frontiera con la Spagna.

Il weekend successivo, quello dell'Atto II, è contrassegnato dalla presenza di 106.000 manifestanti mobilizzati sabato 24 novembre a livello del Paese, dove si registrano oltre 1600 iniziative effettuate dai Gilets Jaunes in varie località del territorio nazionale. A Parigi circa 8.000 persone partecipano alla mobilitazione che interessa in particolare luoghi altamente simbolici della capitale. I Gilets Jaunes si radunano in particolare nella zona dell'Avenue des Champs Élysées, che sorge in un'area prossima tanto alla sede dell'Assemblée Nationale, la Camera dei deputati, quanto dell'Élisée, il palazzo presidenziale. Una zona dove la manifestazione non era stata autorizzata. Lanci di lacrimogeni da parte delle forze dell'ordine fungono da catalizzatore per la creazione di barricate da parte di manifestanti. Una situazione di scontro che favorisce anche l'attività di casseurs, gruppi non precisamente identificati di persone che creano danni soprattutto a installazioni commerciali presenti nell'area. In altre località del Paese le mobilitazioni si svolgono pacificamente: a Lille sfilano circa 600 persone nel centro della città capoluogo del Dipartimento del Nord e della regione Hauts-de-France; a Rennes - capoluogo del Dipartimento d'Ille-et-Vilaine e della Regione Bretagna- la principale iniziativa dei Gilets Jaunes consiste nell'apertura di un casello che rende gratuito il pedaggio autostradale; a Lione - capoluogo dell'omonima area metropolitana e della Regione Auvergne-Rhône-Alpes - si svolge una manifestazione che sfila nel centro cittadino; una manifestazione si svolge nella calma anche a Toulouse, capoluogo dell'omonima area metropolitana, del Dipartimento della Haute-Garonne e della Regione Occitania; lo stesso si verifica a Montpellier; infine, per concludere sulle azioni tenute nei centri di maggiore importanza, a Marsiglia una delle azioni più significative è svolta nell'area di un centro commerciale, dove i manifestanti intervengono sulla 
regolazione dei flussi del traffico automobilistico e spiegano ad altri cittadini le ragioni della loro azione.

Queste manifestazioni - i network relazionali che permettono di organizzarle e di discuterne i significati che gli attori stessi vi attribuiscono definendo gli obiettivi da perseguire - costituiscono, insieme all'intensificarsi della comunicazione online, anche il contesto dialogico in cui i Gilets Jaunes elaborano, a cominciare dalle prime settimane di mobilitazione, le loro piattaforme rivendicative. In questo stesso contesto e a seguito di un sondaggio condotto online - a cui, stando alle affermazioni degli stessi attori che lo promuovono, partecipano circa 30.000 persone $^{7}$ - è messa a punto dagli attori delle mobilitazioni una lista di "Direttive del popolo" da presentare agli eletti dell'Assemblée Nationale" (Camera dei deputati), a cui viene inoltrata, in forma imperativa, richiesta di loro traduzioni in leggi ${ }^{8}$.

L'Atto III, avviato il primo dicembre, vede la partecipazione di circa 136.000 persone a livello del Paese. Le concentrazioni di Gilets Jaunes sono contrassegnate a Parigi da scontri violenti tra manifestanti e forze dell'ordine nell'area degli Champs-Elysées o in spazi adiacenti. In seguito a questi scontri oltre 100 persone rimangono ferite e le forze dell'ordine procedono a oltre 400 fermi. Nella stessa Parigi si segnalano quasi 200 inizi di incendio. Altri scontri, in cui si registra la presenza di casseurs, si hanno a Bordeaux, Saint-Etienne e a Strasburgo. Mentre le manifestazioni si svolgono senza incidenti in altre parti del Paese.

Le altre mobilitazioni che hanno luogo nel mese di dicembre vedono da un lato diminuire il numero dei manifestanti con l'approssimarsi delle feste natalizie e di fine anno e da, un altro lato, apparire con maggiore insistenza problematiche di ordine politico che si congiungono alle questioni culturali e sociali affrontate con l'inizio delle mobilitazioni.

${ }^{7}$ FranceBleu (2018), ???????.

${ }^{8}$ Le "Direttive del popolo" da tradurre leggi riguardano: «l'eliminazione della condizione di senza fissa dimore (Zéro Sdf); l'incremento dei fattori progressivi dell'imposta sul reddito; l'aumento dello Smic (cfr. par. 1); favorire lo sviluppo del piccolo commercio; un grande piano per l'isolamento termico degli immobili; far rimanere il sistema delle pensioni definito in base a principi solidaristici e dunque socializzato; fine dell'aumento delle tasse sul carburante; nessuna pensione deve essere inferiore a 1.200 euro; tutti i rappresentanti eletti debbono percepire il salario medio, le loro spese di trasporto dovranno essere sorvegliate e rimborsate solo dietro presentazione di giustificativi, essi avranno diritto a buoni pasto e a buoni vacanze; i salari di tutti francesi, come anche le pensioni, devono essere indicizzati rispetto all'inflazione; proteggere l'industria francese e vietare le delocalizzazioni; porre fine al distacco di lavoratori (travail détaché) regolato anche da normative EU oltre che nazionali; garantire la sicurezza occupazionale» (Gilets Jaunes, Nov. 2018, Directives du Peuple, pdf, ??????). 


\section{Culturale, sociale, politico}

Le mobilitazioni dei Gilets Jaunes ricevono un'importante copertura mediatica. Le edizioni online o a stampa di importanti quotidiani - di differenziato orientamento culturale e politico - di rilievo nazionale - che vanno da Le Figaro a Le Monde, da Le Parisien Aujourd'hui en France a Libération e a La Croix - come anche di stampa regionale e locale - quali la Voix Du Nord e Ouest-France - coprono ampiamente gli eventi e i dibattiti riguardanti queste mobilitazioni. Lo stesso avviene con le reti televisive pubbliche o private come France 2, Tfi o France 3 e, in particolare, con Bfm TV fortemente impegnata nel coprire gli avvenimenti e nel proporre analisi o dibattiti su queste azioni. Ampie coperture e analisi che riguardano le mobilitazioni sono, inoltre, assicurate anche da emittenti radio nazionali e locali.

Una parte di queste strutture mediatiche presenta l'azione collettiva con connotazioni critiche, un'altra parte invece le rappresenta in termini interlocutori, se non di appoggio. Un'azione che inizialmente si costituisce soprattutto criticando le politiche vigenti in campo economico o sociale perseguite dal governo in carica e sostenute da funzionari delle tecnostrutture dell'apparato di Stato. Politiche che prendono senso puntando a perseguire prospettive di sviluppo economico e sociale proprie di visioni liberiste della finanza e dell'economia ${ }^{9}$, visioni a cui, da fine XX secolo, si riferiscono, per formulare le loro politiche economiche e sociali, non solo gli esecutivi di Francia e di altri importanti Paesi UE, ma anche di altre parti del pianeta. Visioni criticate da studiosi che propongono prospettive alternative di sviluppo economico ${ }^{10}$; da altri che suggeriscono di approcciare la questione dello sviluppo ispirandosi a principi di giustizia e di affermazione dei diritti dei diritti dell'uomo, inteso come soggetto che ha il diritto di controllare l'evoluzione della propria esistenza ${ }^{11}$; e, da altri ancora, che insistono sull'introduzione di una tassazione a livello mondiale sui flussi di capitale ${ }^{12}$ e una ridefinizione dell'imposta progressiva sul reddito, non solo per promuovere la riduzione delle diseguaglianza sociali attraverso l'intervento fiscale, ma anche per favorire ulteriormente la razionalizzazione dello sviluppo economico ${ }^{13}$.

9 N. Friedman (2002), Capitalism and Freedom. Fortieth Anniversary Edition, University of Chicago Press, Chicago.

10 J.E. Stiglitz (2012), The Price of Inequality. How Today's Divided Society Endangers Our Future, W.W. Norton \& Company, New York.

11 A. Sen (2010), The Idea of Justice, Penguin Books, New York.

${ }_{12}$ T. Piketty (2013), Le capital au XXI siècle, Seuil, Paris, pp. 835-882.

${ }^{13}$ Ivi, pp. 793-834 
Rappresentazioni critiche delle situazioni sociale ed economica di rilievo globale oppure nazionale e locale che trovano riscontri nel linguaggio contestativo dei Gilets Jaunes, improntato a denunce delle ingiustizie e a rivendicazioni di giustizia sociale o di giustizia fiscale. Denunce e rivendicazioni i cui contenuti, stando ai sondaggi di opinione, sono valutati favorevolmente da buona parte dell'opinione pubblica nazionale. La maggioranza dei francesi intervistati lungo il periodo di svolgimento delle mobilitazioni dichiara infatti di sostenere o di vedere con simpatia le mobilitazioni dei Gilets Jaunes. Una maggioranza che decresce per corso del tempo, passando da un consenso che si aggira intorno al 70\% registrato in occasione dei primi Atti di novembre e dicembre 2018, per assestarsi intorno al 50\% in occasione degli Atti del febbraio dell'anno successivo. Risulta invece in aumento la minoranza degli intervistati che si oppone o si dichiara ostile alle mobilitazioni passando dal $22 \%$ circa registrato nel corso dei primi atti, all'oltre 30\% registrato nel corso degli ultimi. Rimane, infine, quasi costante, la percentuale di coloro che affermano di essere indifferenti rispetto alle iniziative dei Gilets Jaunes, oscillando tra il 12\% riscontrato al tempo delle prime mobilitazioni il $16 \%$ delle ultime rilevazioni ${ }^{14}$.

L'impatto mediatico e il relativo consenso, rilevato dai sondaggi, dell'opinione pubblica nazionale rispetto ai temi sollevati dalle mobilitazioni, porta nelle stesse fila dei Gilets Jaunes a maturare la consapevolezza del rilievo politico che le loro iniziative ricoprono. Un rilievo che si palesa in presenza di una situazione istituzionale diventata difficile con la crisi che investe i partiti tradizionali ${ }^{15}$.

${ }^{14}$ Secondo i risultati di sondaggi svolti in fasi preparatorie, in considerazione di eventi salienti (per es. danni all'Arc de Triomphe, moratorie sull'amento delle tasse annunciate dal governo, sfondamento della porta di un ministero da parte di manifestanti) o in seguito allo svolgimento degli atti, coloro che si dichiarano favorevoli o sostenitori delle mobilitazioni costituiscono, rispetto al totale degli intervistati, il 71\% il 6-7 nov. 2018, il 69\% il 13-14 nov., il $66 \%$ il $20-21$ nov. 2018 , il $71 \%$ il $27-28$ nov. 2018 , il $72 \%$ il $3-4$ dic. 2018 , il $66 \%$ il 5-6 dic. 2018, il 68\% 11-12 dic. 2018, 57\% l'8-9 gen. 2019, il 58\% il 15-16 gen. 2019, il 58\% il 22-23 gen. 2019 , il 58\% il 29-30 gen. 2019, il 55\% il 5-6 feb. 2019, il 50\% il 13-14 febbraio dello stesso anno. Gli intervistati che dichiarano di opporsi o di essere ostili alle mobilitazioni sono l'11\% il 6-7 nov. 2018, il 15\% il 13-14 nov., il 22\% il 20-21 nov. 2018, il 19\% il 27-28 nov. 2018, il 20\% il 3-4 dic. 2018, il 24\% il 5-6 dic. 2018, il 24\% 11-12 dic. 2018, il 31\% l'8-9 gen. 2019 , il $27 \%$ il $15-16$ gen. 2019 , il $26 \%$ il $22-23$ gen. 2019 , il $26 \%$ il $29-30$ gen. 2019 , il $29 \%$ il 5-6 feb. 2019, il 34\% il 13-14 febbraio dello stesso anno. Gli intervistati che si dichiarano indifferenti alle mobilitazioni sono il 18\% il 6-7 nov. 2018, il 16\% il 13-14 nov., il 12\% il 20-21 nov. 2018, il 10\% il 27-28 nov. 2018, 1'8\% il 3-4 dic. 2018, il 10\% il 5-6 dic. 2018, 1' 8\% 11-12 dic. 2018 , il 12\% l' $8-9$ gen. 2019 , il 15\% il 15-16 gen. 2019, il 16\% il $22-23$ gen. 2019 , il $16 \%$ il 29-30 gen. 2019, il 16\% il 5-6 feb. 2019, il 16\% il 13-14 febbraio dello stesso anno («Le regard des Français sur les Gilets Jaunes», Ifop - Le Journal du dimanche, Février 2019, pdf, ?????.).

${ }^{15}$ Una crisi che coinvolge il maggiore partito della sinistra, il Parti Socialiste, come anche le formazioni tradizionali della destra repubblicana seriamente penalizzate in occasione delle 
In questo contesto emergono presso gli stessi manifestanti esigenze di perseguire obiettivi politici per incidere sui rapporti di forza che emergono nella strutturazione della vita sociale. Esigenze che si declinano principalmente in due prospettive. La prima si declina puntando alla costruzione di spazi istituzionali che abbiano affinità con pratiche di democrazia deliberativa, il che si traduce nella richiesta, proveniente dalle manifestazioni, e rivolta all'esecutivo, dell'istituzione dei Ric (Referendum d'initiative citoyenne referendum di iniziativa popolare), intesi come consultazioni referendarie che permettano di far affermare la presenza attiva dei cittadini nella formulazione di leggi, o altre normative, e nella presa delle decisioni politiche ${ }^{16}$.

Il referendum è in questo modo inteso presso gli attori che promuovono questa iniziativa, come espressione della volontà dei singoli e di loro incidenza diretta, senza delega, sulle scelte politiche da operare. Un'intenzione di sperimentare forme di democrazia diretta che si connette per un verso all'esperienza maturata dai Gilets Jaunes con la comunicazione online - priva tanto di forme di gestione affidata o conquistata da una oppure da molteplici aziende specializzate nella gestione dei flussi di informazione, quanto di agenti regolatori dei dibattiti e dei confronti fra i singoli fluidi promotori o partecipanti alle azioni svolte in comune - e, per un altro, alla consapevolezza che il perdurare delle loro mobilitazioni li coinvolge come soggetti individuali non deleganti, soggetti che controllano l'evoluzione della pro-

elezioni presidenziali del 2017 che vedono il centrista Emmanuel Macron battere al secondo turno Marine Le Pen candidata della formazione post-fascista Front National (A.L. Farro, 2014, «Movimenti collettivi e forze populiste del XXI secolo», in F. Antonelli, E. Rossi, a cura di, Homo dignus. Cittadinanza, democrazia e diritti del mondo in trasformazione, Cedam, Padova, pp. 109-143), come anche in consultazioni successive locali o nazionali. Una crisi che vede anche l'affermarsi di importanti associazioni politiche come quelle costituite guardando alle presidenziali del 2017, forze come La France Insoumise (Lfi) - costituita nel 2016 con la leadership di Jean-Luc Mélenchon e collocata su un terreno di sinistra radicale e, allo stesso tempo, si schiera, non senza enigmaticità, in difesa della sovranità nazionale rispetto alla UE (Lfi, La France Insoumise, 2018, L'Avenir en commun, en Europe aussi! Programme de La France Insoumise pour les élections européennes 2019, pdf, ?????) - o come la stessa République en Marche di Emmanuel Macron, forza centrista a sua volta fondata nel 2016.

${ }^{16}$ Si tratta di una delle «Direttive del popolo» che i Gilets Jaunes chiedono ai parlamentari dell'Assembée Nationale (Camera dei deputati) di trasformare in legge. Questa direttiva riguarda l'istituzione di un «Referendum popolare da inserire nella Costituzione» e le modalità di svolgimento di questa consultazione referendaria da realizzare attraverso la «Creazione di un sito leggibile ed efficace, inquadrato da un organismo indipendente di controllo, sito in cui le singole persone (les gens) potranno presentare una proposta di legge. Se questa proposta di legge ottiene 700.000 firme, dovrà essere discussa, completata, emendata dall'Assemblée Nationale che avrà l'obbligo, (un anno giorno per giorno dopo l'ottenimento delle 700.000 firme) di sottometterla al voto dell'insieme dei francesi» (Gilets Jaunes, Nov. 2018, Directives du Peuple, cit.). 
pria esistenza anche sul piano del loro inserimento fattivo nei processi di decisione politica.

La seconda prospettiva, perseguita - come si vedrà in seguito nel testo (cfr. ?????) - da una parte degli attivisti Gilets Jaunes, consiste nel tentativo di costituire un'entità politica in grado di presentarsi con proprie liste alle consultazioni elettorali a vari livelli, al fine di avere una propria rappresentazione del sistema istituzionale.

Le mobilitazioni che seguono le iniziative viste in precedenza (cfr. 4), sottolineano con maggiore decisione il rilievo politico che comporta l'azione collettiva condotta in Francia, dove il sistema istituzionale, come avviene anche in altri stati UE e Paesi occidentali, deve affrontare nuove sfide di ristrutturazione istituzionale.

L'Atto IV delle mobilitazioni, svolte nel weekend che ha inizio l'8 dicembre, fanno registrare ancora una partecipazione significativa. In tutto il Paese fonti ministeriali segnalano la presenza di 136.000 manifestanti che sfilano, oltre che, in 10.000, a Parigi, anche in altre grandi città come Nantes, Bordeaux, Marsiglia, Nizza, Toulouse e Montpellier. Mobilitazioni si hanno anche in centri minori e si segnalano rallentamenti o blocchi della circolazione nelle aree urbane o su percorsi autostradali, come avviene su una delle carreggiate del tratto dell'A62 che collega Bordeaux a Toulouse, dove si registra la presenza di manifestanti. Si verificano, infine, anche diversi episodi di scontri con le forze dell'ordine nel corso dello svolgimento di quest'insieme di manifestazioni che continuano ad affrontare questioni di ordine culturale, sociale ed economico, ma anche tematiche politiche, intervenendo in particolare sul Ric.

Le mobilitazioni sono, con 66.000 manifestanti presenti a livello del Paese, in calo nel corso dell'Atto V che si svolge nel weekend iniziato il 15 dicembre. Nel periodo delle feste natalizie, con 1'Atto VI del weekend iniziato il 22 dicembre, la partecipazione risulta essere, con 38.600 manifestanti mobilitati a livello del Paese, ancora inferiore a quella registrata nella settimana precedente. Una riduzione confermata durante l'Atto VII che ha inizio il 29-12-2018 e vede la partecipazione di 32.000 manifestanti. Mentre la partecipazione comincia a re-intensificarsi con le mobilitazioni che si hanno a Parigi, in altre aree urbane e differenti zone dopo le feste di fine anno. Mobilitazioni in cui si registra la presenza di 50.000 manifestanti nell'Atto VIII del weekend iniziato il 5-01-2019, di 84.000 nel corso dell'Atto IX del fine settimana successivo, sempre di 84.000 nell'Atto X del weekend iniziato il 19-01-2019, di 69.000 nell'Atto XI del weekend successivo e di 58.600 partecipanti alle manifestazioni dell'Atto XII che ha inizio il sabato 2 febbraio del nuovo anno. L'Atto XIII del weekend iniziato il 9 febbraio vede 
la partecipazione 51.400 in tutto il Paese e di 4.000 a Parigi. L'Atto XIV, avviato il 16 febbraio, vede la partecipazione di 41.000 manifestanti, con 5.000 che sfilano nella capitale. L'Atto XV che inizia il 23 febbraio, vede la partecipazione di 46.600 manifestanti, di cui 5.800 a Parigi e i restanti in altri centri e località del Paese ${ }^{17}$.

Nel corso di queste manifestazioni sono frequenti gli scontri tra manifestanti e forze dell'ordine. In diverse occasioni gruppi di casseurs diventano con le loro azioni parte significativamente visibile delle mobilitazioni. A Bordeaux, il 09-12-2018, nel corso del Atto IV, a fine prima giornata si hanno scontri tra forze dell'ordine e gruppi radicali di manifestanti - designati come casseurs da altri partecipanti a cortei tenuti nel centro cittadino- che appiccano il fuoco in parti dei percorsi viari, danneggiano arredi pubblici, assaltano e svaligiano negozi. Si hanno anche episodi di attacchi a luoghi simbolici del potere, come avviene a Rouen, in Normandia, dove nel corso della manifestazione del 29/12/2018, al tempo dell'Atto VII, viene appiccato il fuoco alla porta della sede locale della Banque de France. A Parigi, per fare un altro esempio, il 0501-2019, nel corso dell'Atto VIII, una decina di manifestanti si appropriano di un muletto presente in strada e sfondano la porta di accesso dell'immobile dove ha sede il Ministère des relations avec le Parlement, evento considerato molto grave da parte di rappresentanti del governo. A Place de la Bastille, a Parigi, mentre il 26 gennaio si svolgeva la mobilitazione dell'Atto XII, un manifestante, una delle figure simbolo dei Gilets Jaunes, viene ferito gravemente a un occhio dallo scoppio di un esplosivo lanciato dalle forze dell'ordine. Nel corso dell'Atto XIII, iniziato il 9 febbraio, nella capitale un manifestante perde una mano con cui aveva raccolto un lacrimogeno lanciato dalle forze dell'ordine su partecipanti a un corteo. Nel corso dell'Atto XIV, avviato il 16 febbraio a Parigi, a margine di una manifestazione, viene aggredito da un manifestante verbalmente (con frasi antisemite come "Barre toi sale sioniste de merde, grosse merde sioniste, nous sommes le peuple, la France elle est à nous") il filosofo e noto studioso Alain Finkielkraut. Episodio fortemente criticato da esponenti politici del governo e dell'opposizione, da intellettuali e opion leader come anche all'intero delle fila degli stessi Gilets Jaunes. Ma anche episodio che concorre a rilanciare il dibattito e le denunce su nuove manifestazioni di antisemitismo che investono la Francia e l'Europa nel suo insieme.

17 Tutti i dati sul numero dei partecipanti alle mobilitazioni qui riportati sono ripresi dalle informazioni diramate dal Ministère de l'Intérieur. Fonte a cui, non potendo ricorrere a tutte le altre per motivi di spazio, mi sono riferito pur sapendo che gli stessi Gilets Jaunes, diversi osservatori, esponenti del sindacato di polizia, rappresentati politici dell'opposizione e altri attori ne contestano la veridicità valutando il numero dei manifestanti superiore a quello indicato dal Ministero. 


\section{I "ronds-points"}

Le mobilitazioni dei Gilets Jaunes assumono anche un'altra forma con l'occupazione di aree in cui sono presenti ronds-points che consistono rotatorie installate su parti di tratti stradali al fine di favorire lo scorrimento del traffico automobilistico. Una forma di lotta che interessa diverse aree del territorio nazionale e che è concomitante con le azioni condotte online e con le mobilitazioni che danno vita agli Atti ritmati dalle manifestazioni tenute nel corso dei weekend. A differenza di queste manifestazioni che si svolgono con scadenza settimanale, i presidi dei ronds-points sono insediamenti che si vogliono stabili e che sono animati dalla presenza, in molti casi continuativa, di Gilets Jaunes impegnati nel testimoniare in questo modo le ragioni della loro lotta, a spiegarne a passanti e automobilisti il senso che vi attribuiscono, a imporre sovente un loro modo di regolamentare il traffico, non necessariamente gradito ai conducenti di auto e di altri veicoli che circolano sulle rotatorie.

Queste occupazioni sono oggetto di interventi istituzionali che puntano a stopparne $\mathrm{o}$, almeno, a contenerne la diffusione. Nelle seconda metà di dicembre fonti governative danno notizia dello smantellamento di circa 170 occupazioni, mentre intorno a 360 risultano essere ancora attive. Non mancano, però, anche forme di resistenze esplicite allo smantellamento, resistenze che, secondo fonti di emanazione governativa, sono affrontate dagli agenti preposti attraverso il confronto e il dialogo con i manifestanti e facendo ricorso il meno possibile alla forza.

Le rotatorie occupate sono luoghi di presenza simbolica e di manifestazione del dissenso culturale, sociale e politico espresso nei confronti di attori dirigenti, élite e potere politico cui sono imputate ingiustizie sociali, dominio ed estraneità dai problemi dei cittadini della "petite classe moyenne" strati meno elevati e delle classi popolari. Le prime installazioni dei presidi su spazi in cui sono installate rotatorie si hanno nel mese di novembre, sulla scia delle manifestazioni che si svolgono, come visto, in varie parti del Paese.

L'intervento governativo di smantellamento di queste installazioni si profila mentre una parte rilevante dei Gilets Jaunes impegnati in questo tipo di mobilitazione punta invece a rafforzarla. Una resistenza che sovente si esplicita con azioni di ricostruzione dell'impianto laddove questo viene distrutto o smantellato in seguito a provvedimenti amministrativi, per intervento delle forze dell'ordine o per altri motivi. È quanto si verifica a Montceau-Les-Mines, vecchio centro minerario e industriale - centro situato nel Dipartimento della Saune-et-Loire della regione Borgogna-Franche-Comté - divenuto, dopo il completamento della chiusura definitiva di tutte le attività estrattive che ha luogo a fine anni Novanta-inizi Duemila, un territorio contrassegnato 
in larga parte da disoccupazione e destrutturazione della vita sociale. I principali protagonisti dell'installazione del presidio sono direttamente coinvolti in questo processo di destrutturazione che ha investito non solo i lavoratori, ormai anziani, colpiti dallo smantellamento delle miniere e dalla crisi delle attività industriali, ma anche persone, donne e uomini di diversa età, formazione e condizione professionale che condividono situazioni di precarietà occupazionale, bassi salari, precarietà di reddito o di disoccupazione.

Il presidio di Montceau-Les-Mines è installato dai Gilets Jaunes nell'area del rond-point du Magny, nei pressi del tracciato della Rcea - Route Centre Europe Atlantique, un importante tracciato stradale gratuito e molto frequentato che attraversa la Francia da Est a Ovest - al fine di rendere il più possibile visibile la loro iniziativa. I manifestanti sono donne e uomini, giovani e anziani. Sono operai o discendenti da famiglie di operai, impiegati, lavoratori in proprio o disoccupati che si sentono convolti in una lotta di rivendicazione di diritti sociali come anche di affermazione e riconoscimento della propria dignità.

Il loro presidio iniziale è costruito in uno spazio delimitato dal circuito della rotatoria e consiste in installazioni simili a baracche equipaggiate con utensili, vettovaglie e postazioni di riposo. Uno spazio che è in parte delimitato da pneumatici messi a precaria protezione del luogo in cui si hanno discussioni tra quanti sono impegnati nella mobilitazione.

Gli orientamenti politici di quanti sono impegnati con continuità nel presidio e di coloro che vi circolano sono molteplici. Vi sono persone, operai, che si pongono il problema di votare per il Rassemblement National (RS) - il partito fondato e guidato da Marine Le Pen, mantenendo le connotazioni culturali e politiche post-fasciste del Front National $(\mathrm{FN})^{18}$, formazione politica che questa stessa leader politica contribuisce a dissolvere in seguito alla sconfitta subita nel 2017 alle elezioni presidenziali - da loro ritenuta una personalità politica attenta ai problemi delle classi popolari e non necessariamente ostile all'immigrazione, in quanto intende espellere dal Paese solo i migranti irregolari senza documenti. Altri si sentono invece vicini alla formazione La France Insoumise (Lfi).

Le occupazioni dei ronds-points si declinano in questo modo come iniziative critiche condotte dai Gilets Juanes per esprimere il loro dissenso rispetto alle élite dirigenti e al dominio che queste esercitano sulla società condizionando gli sviluppi della vita individuale di singoli cittadini. Riunirsi e insediarsi nelle aree delle rotonde significa denunciare l'ingiustizia sociale cui è esposta una parte rilevante della popolazione e di cui i singoli

18 A.L. Farro (2014), "Movimenti collettivi e forze populiste del XXI secolo", cit. 
manifestanti si sentono individualmente -affermando di non vergognarsi di dichiararlo - componente costitutiva. Manifestare presidiando i ronds-points significa anche asserire di avere il diritto a non rimanere in questo stato di sottomissione. Come significa anche affermare la fierezza di sentirsi cittadini "à part entière", di voler essere singolarmente soggetti decisori diretti della definizione dei programmi e delle scelte politiche da operare per pervenire alla giustizia sociale.

L'azione collettiva dei singoli manifestanti di Montceau-Les-Mines si spiega, di conseguenza, come affermazione del diritto individuale alla giustizia sociale. Un diritto che si combina col rifiuto di veder condizionato lo sviluppo della propria esistenza dalle politiche imposte dalle élite e dal dominio che esercitano sulla vita sociale. Il coinvolgimento individuale nell'azione collettiva si presenta come un processo di soggettivazione perseguito dal singolo intenzionato a divenire controllore dello sviluppo della propria esistenza sottraendosi, anche se per un tempo limitato, al dominio delle élite ${ }^{19}$. Una soggettivazione che diviene però negativa quando il tentativo di sottrarsi al potere delle élite e degli apparati delle tecnostrutture, si combina con la negazione del riconoscimento dello stesso diritto ad altri, ai singoli migranti in particolare. L'azione collettiva dei Gilets Jaunes si profila in questo modo nella duplice e contraddittoria versione di un'azione contrassegnata per un verso dalla soggettivazione e, per un altro, dalla soggettivazione negativa che, a sua volta, si combina con propensioni politiche contrassegnate come fasciste o di estrema destra.

Azione collettiva che trova una sua traduzione pratica non solo nella mobilitazione di Montceau-Le-Mines, ma anche in altre, distinguibili principalmente in due tipologie. Due realtà d'azione tra loro diverse che si distinguono principalmente per le modalità adottare nella gestione dei presidi e nel rapporto che i manifestanti stabiliscono con quanti attraversano, soprattutto in macchina o altri veicoli a motore, i circuiti viari dell'area della rotatoria occupata.

Modalità d'azione che si ritrovano in due occupazioni di ronds-points che hanno luogo in una stessa area del territorio di Sens, un comune a circa 100 km a Sud-Est di Parigi, situato nel Dipartimento dell'Yonne della Regione Borgogna-Franche-Comté.

Il primo insediamento, a cui i manifestanti attribuiscono il nome di "rondpoint des Gaulois", è dovuto a decine tra artigiani, casalinghe, operai e disoccupati, donne e uomini con età che varia dalla ventina a oltre la sessantina

19 A.L. Farro (2014), «A New Era for Collective Movements: The Subjectivation of Collective Action», in A.L. Farro, ?? Lustiger-Thaler (eds.), Reimaging Social Movements. From Collective to Individuals, Ashgate, Burligton, pp. 15-34. 
d'anni, i quali stabilmente o a rotazione assicurano l'occupazione del luogo. Questi occupanti affermano di avere una posizione di ferma opposizione al potere delle élite e alla politica governativa, di cui viene individuato come principale responsabile il presidente Emmanuel Macron, e di rivendicare un radicale cambiamento di queste stesse politiche a favore dei cittadini della "petite classe moyenne" e delle classi popolari, soprattutto se sono cittadini i cui insediamenti abitativi sono collocati in aree distanti dai servizi e dalle infrastrutture dei medi e grandi centri urbani.

Questi stessi attori tengono inoltre a sottolineare il fatto che il loro insediamento nell'area della rotonda si caratterizza per essere pacifista. Nel senso che gli occupanti non hanno e non intendono avere comportamenti aggressivi, non creano danni materiali nel luogo del loro insediamento, non accettano la presenza tra loro di persone propense all'alcolismo, non aggrediscono automobilisti di passaggio imponendo loro di indossare il gilet giallo e non rallentano o bloccano la circolazione sulla rotonda. Questi attori intendono con la loro mobilitazione semplicemente esprimere un messaggio che denuncia la situazione socio-economica in cui versano e che pone l'accento sulla loro esigenza di ottenere politiche che permettano un radicale miglioramento della loro situazione. Un messaggio con cui perseguono anche l'obiettivo di sensibilizzare gli abitanti dell'area spiegando con cortesia le ragioni della loro lotta chiedendo anche di sostenerla.

Un modo di intendere, di sostenere e di partecipare alla mobilitazione dei Gilets Jaunes con modalità d'azione che differiscono molto da quelle adottate dagli occupanti insediati in un'area vicina, presso un esercizio della catena Auchan. Sono altre modalità d'azione che caratterizzano una seconda componente delle mobilitazioni dei ronds-points. I manifestanti di questo secondo insediamento esprimono critiche verso le élite e le forze governative simili a quelle su cui insistono gli attori del primo. Simili sono anche le loro rivendicazioni di carattere socio-economico e politico. Ma a differenza dei loro vicini dell'insediamento visto in precedenza, si preoccupano di dare visibilità alla loro azione intervenendo sulla gestione del traffico di automobili e di altri veicoli che attraversano la rotonda, creano rallentamenti della circolazione, esprimono forte dissenso verso autisti di automobili e conduttori di altri veicoli che non indossano gilet gialli inducendoli a farlo, intervenendo con aggressività verso coloro che resistono al loro invito e sui veicoli di questi ultimi. Questi occupanti sono inoltre poco attenti a mantenere il decoro del luogo del loro insediamento e non disdegnano di avere condotte che vi creano danneggiamenti.

Le occupazioni dei ronds-points presentano in questo modo le stesse principali modalità d'azione che si hanno con le mobilitazioni offline che hanno 
luogo con le manifestazioni tenute nei weekend a Parigi e altre città o località del Paese. Nel corso di quelle mobilitazioni una parte dei manifestanti intende esprimere la propria opposizione alle politiche governative sfilando pacificamente per le strade cittadine, mentre altri adottano condotte più radicali che si imbattono con gli interventi repressivi delle forze dell'ordine. Altri ancora, cui viene attribuita correntemente la denominazione di casseurs, si dedicano, a loro volta, alla distruzione di parti degli arredi o delle strutture urbane, all'assalto a vetrine di negozi -a volte anche per sottrarre le merci esposte - a installazioni simbolo di poteri economici - come bancomat o le sedi di banche, compresa la Banque de France - o politici, come le sedi di organismi istituzionali, che vanno dai comuni fino all'Assemblée Nazionale, la Camera dei deputati.

Tutte queste modalità d'azione sono parti costitutive delle mobilitazioni dei Gilets Jaunes, che rappresentano una delle più lunghe, in termini temporali, azioni collettive di portata sociale, culturale e politica che investono la Francia nel corso di questo secolo e di quello precedente. Un'azione collettiva che: 1) si costituisce criticando e rivendicando innanzitutto alternative alle misure fiscali introdotte dal governo con l'aumento della tassa sul carburante e, in termini più complessivi, giustizia fiscale e giustizia sociale; 2) si struttura principalmente con iniziative online, manifestazioni che danno vita agli Atti con scadenza settimanale, presidi di ronds-points, blocchi temporanei di tratti di strade e autostrade; 3 ) si oppone al potere di tecnostrutture proprie di élite nazionali e di forze della finanza globale; 4) persegue obiettivi di miglioramento delle condizioni economiche e sociali degli strati più bassi della classe media ("petite classe moyenne") e delle classi popolari; 5) critica l'accentramento del potere politico nazionale e persegue l'affermazione, soprattutto attraverso il Ric, dell'intervento diretto dei cittadini nella presa delle decisioni politiche.

\section{I luoghi}

Gli spazi in cui prendono avvio le mobilitazioni offline sono costituiti principalmente da un lato da zone periferiche di grandi aree metropolitane, dove risiedono molti pendolari, come per esempio la Seine-et-Marne nella regione parigina, e da aree centrali del comune capoluogo, in questo caso la stessa capitale dove hanno luogo le manifestazioni con maggiori partecipazione e ripercussione mediatica. Anche altre iniziative conflittuali, come le occupazione di ronds-points e le manifestazioni organizzate in aree di centri commerciali, hanno inoltre luogo nella stessa regione parigina e in diverse 
zone di altre grandi aree metropolitane. Da un altro lato, gli spazi interessati dalle mobilitazioni sono costituiti da località di ampie zone - come la valle del Rodano, della Garonna, della Senna e di parte di quella della Loira - attraversate da grandi assi di circolazione, di cui si servono residenti di luoghi densamente abitati per raggiungere, partendo dalla loro abitazione e percorrendo diversi $\mathrm{km}$ per lo più con propri veicoli da alimentare con il carburante necessario, luoghi di lavoro, scuole dei figli, sedi di amministrazioni, servizi sanitari o di altro tipo ${ }^{20}$.

Una parte delle prime manifestazioni dei Gilets Jaunes ha luogo, più in particolare, per un terzo in comuni (228) con meno di 5.000 abitanti e per il $42 \%$ in altri (291) che ne hanno tra 5.000 e 20.000 , allorquando vi sono, tra gli altri comuni interessati, una parte (116) che ne annovera tra i 20.000 e 50.000 e un'altra (65) che, a sua volta, ha oltre 50.000 residenti (Fourquet, Manternach, 2018).

Le mobilitazioni dei Gilets Jaunes hanno del resto come protagonisti anche residenti di piccoli e medi centri urbani situati principalmente in zone rurali o in aree urbane distanti dal centro delle grandi metropoli. Sono soprattutto zone e aree investite da difficoltà economiche e dalla disoccupazione che non è affrontata dall'insorgere di nuove opportunità occupazionali. Si tratta della "Francia periferica", di territori investiti dalla deindustrializzazione, dalla crisi dell'agricoltura, dalla sparizione dei negozi e dei circuiti commerciali, dei servizi amministrativi, sanitari e di altro tipo ${ }^{21}$. Sono anche realtà territoriali che sono male o poco servite da reti di trasporti pubblici, oppure non lo sono per nulla. Di conseguenza, i cittadini che vi risiedono sono obbligati, ancor più che in altri luoghi, a ricorrere a propri mezzi di trasporto per raggiungere centri urbani con insediamenti di attività commerciali, amministrative e di servizi cui è necessario ricorrere per sostenere esigenze di conduzione della vita individuale e familiare.

I tragitti da percorrere con propri veicoli - provvisti preferibilmente di motori diesel alimentati con carburante dal costo inferiore della benzina sono costituiti da strade dipartimentali o nazionali, talvolta non dotate di spartitraffico centrale di separazione tra le due carreggiate di marcia. Automobilisti o trasportatori che viaggiano abitualmente su strade non dotate di questo spartitraffico, hanno ricevuto, dal mese di luglio 2018, multe per eccesso di velocità, avendo superato il limite di $80 \mathrm{~km}$ orari stabilito, come

20 J. Fourquet, S. Manternach (2018), Les “Gilets Jaunes”: révélateur fluorescent des fractures françaises, Fondation Jean Jaurès, Paris, pdf, ??????.

${ }^{21}$ C. Guilluy (2014), La France périphérique: Comment on a sacrifié les classes populaires, Flammarion, Paris. 
visto in precedenza (cfr. par. 2), da nuove normative di sicurezza stradale, introdotte a inizio dello stesso mese di luglio in sostituzione del precedente limite dei $90 \mathrm{~km}$ l'ora. Coloro che le ricevono e altri cittadini che si sentono interessati dal problema criticano l'introduzione di queste sanzioni, ritenendole non confacenti alle esigenze di quanti sono costretti a percorrere frequentemente queste strade, non avendo altri percorsi alternativi da intraprendere e non essendoci nelle aree interessate trasporti pubblici adeguati per assicurare i loro spostamenti. Critiche che si trasformano in argomento di contestazione antigovernativa, diventando uno dei temi principali affrontati dai Gilets Jaunes con l'avvio delle loro mobilitazioni.

I territori della "Francia periferica" costituiscono in questo modo una significativa, rappresentazione spaziale degli squilibri che investono il Paese, interessando, più in particolare, le condizioni di vita di singoli e gruppi di strati popolari o di parte della classe media. Una rappresentazione degli squilibri cui se ne aggiunge un'altra, che prende forma con l'insediamento di classi superiori e medie in aree centrali delle metropoli e di classi popolari in zone lontane dai centri o non urbanizzate. Un fenomeno che si traduce nella "metropotalizzazione" delle ricchezze dei possessori di capitali e delle competenze di quadri superiori, intellettuali o ricercatori. Operai, artigiani e piccoli commercianti al dettaglio si stabiliscono invece nelle aree periferiche $\mathrm{e}$ in quelle dal fragile tessuto urbano prossimo a zone rurali ${ }^{22}$.

\section{Gli orientamenti politici}

Secondo ricerche sociologiche condotte nel corso delle mobilitazioni dei Gilets Jaunes, larga parte dei partecipanti non ritiene in alcun modo pertinente l'eventuale presenza di sindacati e partiti nelle mobilitazioni ${ }^{23}$. I manifestanti intervistati, inoltre, non esprimono orientamenti politici univoci.

${ }^{22}$ H. Le Bras (2014), Atlas des inégalités. Les Français face à la crise, Éditions Autrement, Paris.

${ }^{23} \mathrm{Nel}$ corso delle mobilitazioni del Gilets Jaunes un gruppo di ricercatori di Sciences-Po Bordeaux, dell'Institut National de la Recherche Agronomique (Inra) e del Cnrs avvia una ricerca somministrando questionari ai partecipanti alle manifestazioni. In tutto sono raccolti 700 questionari. I primi risultati che riguardano 166 questionari somministrati durante le manifestazioni dell'Atto II e dell'Atto III, 24 novembre e $1^{\circ}$ dicembre 2018, in sei località (Bordeaux e la sua area, Marsiglia, Caen e la sua area, Rennes, Montpellier, Grenoble e la sua area) fanno rilevare che il $64 \%$ degli intervistati ritiene che i sindacati non debbono svolgere alcun ruolo nelle mobilitazioni e che 1' $81 \%$ considera allo stesso modo il ruolo dei partiti politici (Sciences-Po Bordeaux, Inra, Cnrs, 2018, ????????). 
In maggioranza dichiarano di essere apolitici o di non situarsi né a destra né a sinistra dello schieramento politico. Significativa si rivela però anche la presenza tra i manifestanti di quanti affermano di collocarsi su posizioni di estrema sinistra e di estrema destra. Mentre sono meno rilevanti le presenze di coloro che si situano su posizioni di sinistra, di centro o di destra ${ }^{24}$.

Dalle fila dei Gilets Jaunes insorgono anche tentativi di costruzione di forze politiche capaci di intervenire nel dibattito pubblico e nei luoghi istituzionali di presa delle decisioni politiche presentando le proposte di cambiamento promosse dalle mobilitazioni. Vanno in questa direzione le iniziative prese da Jacline Mouraud - una delle figure simbolo, lo si è visto in precedenza, delle iniziative dei Gilets Jaunes - che si fa promotrice della costituzione di un partito, denominato Les Emergents. Partito che dovrebbe promuovere la trasposizione nel sistema politico di istanze sociali e culturali proprie dei "Gilets Jaunes". L'approssimarsi delle elezioni del parlamento UE previste nel 2019 induce, del resto, anche altri attori delle mobilitazioni a impegnarsi nella costituzione di forze politiche pronte a presentare proprie liste in occasione di questa scadenza elettorale delle mobilitazioni, come avviene con Hayk Shahinyan, uno dei Gilets Jaunes della regione parigina promotore di un raggruppamento politico denominato Mouvement Citoyen.

Dal canto suo, il Pcf, Parti Communiste Français, afferma di condividere buona parte degli obiettivi rivendicativi dei Giltes Jaunes e si dichiara disposto ad accogliere candidati provenienti dalle loro fila nelle proprie liste per le elezioni europee. All'estremo opposto dello schieramento politico, una posizione similare di accoglienza nelle proprie liste per le europee di candidati Gilets Jaunes viene espressa anche dalla formazione politica post-fascista RS, Rassemblement National.

${ }^{24}$ Sempre secondo i dati raccolti dai ricercatori di Sciences-Po Bordeaux, Inra e Cnrs si esplicita da parte degli intervistati diffidenza e distanza rispetto alla politica e, in particolare, verso il sistema rappresentativo. Alla domanda dei ricercatori di situarsi rispetto all'asse destra-sinistra, il numero di relativo maggiore rilievo degli intervistati, il 33\%, afferma di essere apolitica, di non situarsi né a destra né a sinistra. Mentre tra quanti dichiarano di avere un proprio orientamento politico, il 15\% si colloca all'estrema sinistra, il 5,4\% all'estrema destra, il $42,6 \%$ a sinistra, $12,7 \%$ a destra e $6 \%$ al centro (Sciences-Po Bordeaux, Inra, Cnrs, 2018, cit.). Un'altra ricerca condotta dal Collectif Quantité Critique, un gruppo di ricercatori che fa capo all'Università di Lille, perviene a risultati similari. Lo studio riporta i dati di 526 questionari compitati nel corso di un'inchiesta svolta intervistando, tra il 23 e il 30 novembre 2018, Gilets Jaunes in tutto il Paese. Il 51\% dei 526 intervistati rifiuta di collocarsi politicamente a destra o a sinistra. Mentre il 15\% dichiara di essere di sinistra - di questi il 3,6\% si situa all'estrema sinistra - e 1'11,6\% a destra, con il 7,2\% si colloca all'estrema destra (Collectif Quantité Critique, 2018, «Les gilets jaunes ont-ils une couleur politique?», Humanité, 19 décembre). 
L'azione politica proveniente dalle fila dei Gilet Jaunes prende però senso soprattutto con la ricerca di nuovi percorsi da intraprendere per assicurare ai cittadini interventi diretti e vincolanti nei processi di presa delle decisioni politiche. L'esperienza maturata nella comunicazione da quanti promuovono o partecipano alle iniziative online e organizzano, ricorrendo anche a internet, manifestazioni pubbliche come quelle degli Atti o dell'occupazione dei ronds-points, porta questi attivisti a vivere da protagonisti la partecipazione a mobilitazioni che non comportano l'affermazione di leadership stabili. Le stesse figure simbolo di queste mobilitazioni non personificano particolari forme di leadership e non promuovono la costruzione di reali apparati organizzativi stabili per assicurare la continuità dell'azione. La comunicazione online non si costruisce, a sua volta, come sostegno all'affermazione di leadership intese come forze direttive che governano la costruzione di un movimento sociale e politico. I flussi comunicativi si declinano, invece, come strumenti di supporto alla costruzione della democrazia diretta da sviluppare online e senza il controllo di leadership.

L'azione collettiva dei Gilets Jaunes, come accaduto con la costruzione di movimenti sociali quali Occupy Wall Street ${ }^{25}$ si declina, del resto, senza sottostare al controllo di apparati organizzativi e dell'informazione prodotta e controllata da leader attraverso giornali di partito o media similari, come avveniva in precedenza, prima dello sviluppo della comunicazione online ${ }^{26}$. In questo contesto comunicativo, l'implicazione individuale nella costruzione dell'azione collettiva si prefigura come un processo di soggettivazione della stessa azione collettiva, un processo attraverso cui il singolo punta a diventare il controllore della propria storia di vita intendendosi con altri, a loro volta intenzionati singolarmente a seguire un percorso similare, per sottrarsi e perseguire alternative a domini sociali che puntano a modellare e controllare gli sviluppi della vita individuale e sociale. In questo risiede l'abbozzo di progetto politico dei Gilets Jaunes che puntano a ottenere da parte del potere politico l'istituzione di processi di democrazia diretta. La rivendicazione di veder istituire da parte del potere politico i Ric (Referendum d'initiative citoyenne) va in questa direzione.

La risposta presidenziale a queste sfide di ordine politico si declina con il lancio di un "Grand débat national", che si articola con la costituzione di organismi di consultazione politica istituiti a vari livelli territoriali coinvolgendo principalmente eletti locali. Organismi il cui scopo principale consiste

${ }^{25}$ A.L. Farro (2014), ???????, cit.

${ }^{26}$ M. Castells (1996), The Rise of the Network Society, Blackwell, Malden; M. Castells (2012), Networks of Outrage and Hope: Social Movements in the Internet Age, cit. 
nel produrre progetti di intervento politico, economico e sociale in vista della creazione di un nuovo processo di integrazione nazionale da costruire dialogando con le istituzioni dell'Unione Europea, a loro volta messe alla prova dal montare dei populismi.

Si presentano però nelle mobilitazioni dei Gilets Jaunes anche processi di soggettivazione negativa, intesa come negazione dell'altro, di colui che non viene riconosciuto come pari a te e che, come i migranti che possono accedere alle case di edilizia popolare, sono intesi come nemico da annullare.

In questa duplicità della soggettivazione risiede la duplicità della stessa azione dei Gilets Jaunes, che oscilla tra difesa e affermazione di vecchi e nuovi diritti per tutti gli individui e negazione dei diritti di alcuni, come, in particolare, i migranti. Un'oscillazione che sul piano politico si traduce nella propensione di parte dei manifestanti verso posizioni di sinistra e di sinistra radicale, mentre un'altra parte si orienta a destra e all'estrema destra di cui Marine Le Pen rappresenta l'espressione postfascista più significativa.

La costruzione di queste due opposte varianti politiche dell'azione collettiva dei Gilets Jaunes si declina del resto nel contesto di una crisi complessiva dei meccanismi d'integrazione del sistema politico nazionale ${ }^{27}$. Crisi in cui si avverte l'affievolimento, se non la scomparsa, della forza dei partiti tradizionali di destra e di sinistra, dimostrata soprattutto in seguito alle ultime elezioni presidenziali che ne costituiscono la rappresentazione più significativa $^{28}$.

\section{Conclusioni}

Le mobilitazioni dei Gilets Jaunes costituiscono un'esperienza propria di azione collettiva in cui le iniziative online e quelle offline si alimentano a vicenda dando vita ad azioni conflittuali di lunga durata, di ampia ripercussione mediatica di significativo impatto istituzionale.

Presiedono a quest'azione ragioni socio-economiche che riguardano rivendicazioni di giustizia sociale e giustizia fiscale, come anche l'opposizione alle élite del potere a livello nazionale e a quelle della finanza globale. Sul piano politico, l'azione collettiva dei Gilets Jaunes sottolinea per un verso il rilievo assunto dalla crisi del ruolo di rappresentazione di interessi

27 M. Wieviorka (2018), Les "gilets jaunes", quand la France se cabre, OpenEdition,

${ }^{28}$ S. Perrineau (sous la dir. de) (2017), Le Vote disruptif. Les élections présidentielle et législatives de 2017, Presses de Sciences Po, Paris. 
e di orientamenti culturali da parte dei partiti tradizionali e, per un altro, l'insorgere di sperimentazioni politiche declinate intenzionalmente in termini di democrazia diretta. Anche se tra i Gilets Jaunes vi sono anche attori attratti da discorsi e da azioni di forze politiche del populismo post-fascista e anti-sistema. 